\title{
Classification of Malaria Cell Images with Deep Learning Architectures
}

\author{
Ahmet Çinar*, Muhammed Yildirim \\ Computer Engineering, Firat University, Elazığ 23100, Turkey
}

Corresponding Author Email: acinar@firat.edu.tr

\section{https://doi.org/10.18280/isi.250105}

Received: 10 November 2019

Accepted: 3 January 2020

\section{Keywords:}

AlexNet, CNN, deep learning, DenseNet201, Malaria, Resnet50

\begin{abstract}
Malaria is a contagious disease caused by the infection of erythrocytes by Plasmodium parasites, which are transmitted to human by parasitic female anopheles' mosquitoes during feeding. Malaria is a type of infection that can be fatal if left untreated. It is very important to classify malaria virus images quickly and accurately using computer-aided systems. Because there are not enough personnel in each health unit to perform this procedure, traditional methods are both time consuming and open to errors. Once malaria images have been classified, it will be easier to diagnose malaria virus related diseases. Multiple methods have been developed to process large amounts of data. In particular, deep learning methods are frequently used for classification. In this paper, Convolutional Neural Networks (CNN) have been used to classify malaria images as healthy and parasited. Then, medium filter and gauss filter are applied to the original dataset. When classifying malaria data, the highest accuracy rate is achieved in the DenseNet201 architecture with gaussian filtered data of $97.83 \%$. It is observed that the result obtained with the preprocessed data are higher. The application is implemented in the Matlab environment and works independently of the size of the images in the data set.
\end{abstract}

\section{INTRODUCTION}

Malaria is a fatal form of disease caused by parasites transmitted by the bites of mosquitoes. Malaria virus is more common in tropical regions on the world average. Malaria is a major threat to global health, with nearly 200 million cases worldwide and more than 400,000 deaths per year. Therefore, in rural areas where there is a lack of infrastructure and the lack of specialized personnel, information systems can be utilized to a great extent. In addition, since the rate of error in traditional methods is higher, the classification studies developed can be used to alleviate the human burden and help to make the correct diagnosis. Early diagnosis of malaria is of great importance in terms of correct diagnosis and the patient's early recovery process $[1,2]$.

Modern information technologies are of great importance in the fight against such a widespread and deadly disease [3]. In particular, deep learning, which has high success in classifying large amounts of data, is utilized [4].

In the literature, various studies related to malaria virus have been conducted by using different models and architectures of deep learning. Vijayalakshmi et al. Proposed a new neural network model to identify infected malaria parasite using the transef learning approach. They proposed the new neural network model by combining the VGG network and the Support vector machine. They stated that this developed network achieved $93.1 \%$ classification accuracy [5].

In their paper, Delahunt et al. Stated that they propose a deep learning model with the advantages presented by simply visualizing their features and activations. They reported that they present malaria cells with a lower model complexity and achieved a performance rate of $98.61 \%$ [6].

Bibin et al. Reported that they developed a new method for classifying 4100 peripheral blood images, either parasitic or non-parasitic, using the deep belief network. They expressed that they train the proposed deep belief network using contrast separation methods and limited Boltzmann machines. They stated that they obtained an F-score $89.66 \%$, sensitivity $97.60 \%$ and specificity ratio of $95.92 \%$ [7].

Rajaraman et al, Alexnet, Vgg16, Xception, resnet5 and Densenet121 models used in their study. They stated that they determined experimentally the layers of the most appropriate model for feature extraction from the basic data. They stated that the results were statistically confirmed and that trained CNNs were successful for feature extraction [8].

In this paper, CNN architectures ResNet50, AlexNet, GoogleNet, DenseNet201, Vgg19 and Inceptionv3 are used. The networks are trained with original data and test results are obtained. Median and Gaussian filters are then applied to the images and new results are obtained after pretreatment of the data in the same operations [9].

The continuation of the article: In the material and methods chapter, deep learning and the architecture used are explained. In addition, the dataset used for training the model is introduced. The third section contains the application and result section. The last section is devoted to the conclusion and future studies.

\section{MATERIAL AND METHODS}

Deep learning techniques have been applied to medical data in many studies [10].

In this section, most preferred CNN architectures, data set and filters used will be examined. Resnet50 [11], Densenet201 [12], Alexnet [13], Googlenet [14], Inceptionv3 [15] models, 
which are among the $\mathrm{CNN}$ architectures, will be examined. Later, to increase the accuracy rate, median and gaussian filters were applied.

\subsection{Deep learning}

Deep learning allows computers to process and learn data. The biggest feature that distinguishes deep learning models from traditional neural networks is that deep learning models consist of multiple layers. Deep learning goes back to pause in 2012. After the Deep Learning model won the ImageNet competition in 2012, the popularity of deep learning began to increase rapidly. One of the reasons that deep learning has become popular recently is the development of cards with increased processing speed. Increasing amounts of data also increased the tendency to deep learning [16].

In this paper, convolutional neural networks are used. CNNs are one of the most preferred deep learning networks for computer vision applications such as image classification. Cnn networks consist of multiple layers. These layers can be classified as Convolution Layer, Fully Connected Layer, Pooling Layer, Rectified Linear Unit (Relu) Layer, Dropout Layer, Normalization Layer and Softmax Layer [17].

$\mathrm{CNN}$ is primarily trained with network data. When the network is fed with input images, it passes through multiple layers to complete the learning process [18]. Figure 1 shows how the original data is classified. Original images are individually processed with DenseNet201, ResNet50, Alexnet, Vgg19, G oogleNet and Inceptionv3 architectures and then classified as parasite and healthy.

Then, Median Filter and Gaussian Filter were operatived separately to all data in dataset. The structure of Figure 2 is used for the classification of the data obtained.

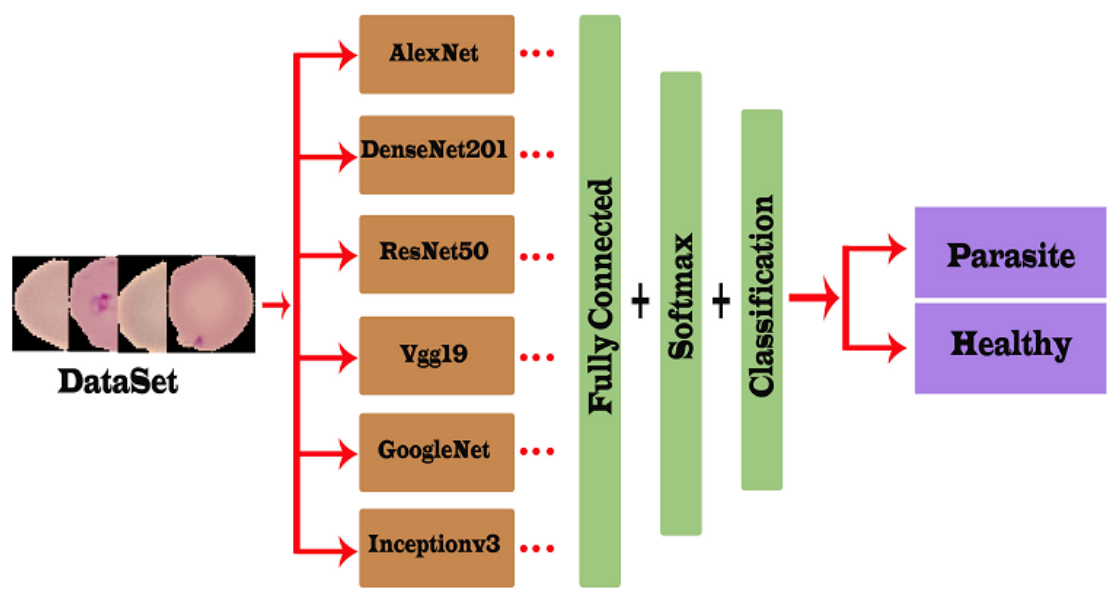

Figure 1. Classification of original data with $\mathrm{CNN}$ architectures

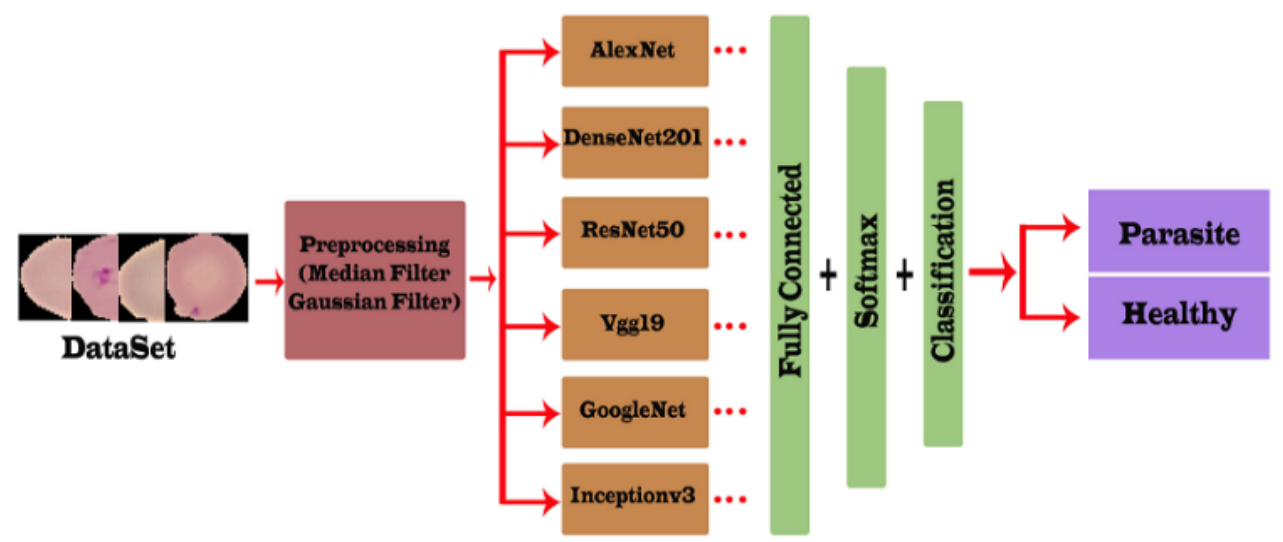

Figure 2. Classification of data after filters with $\mathrm{CNN}$ architectures

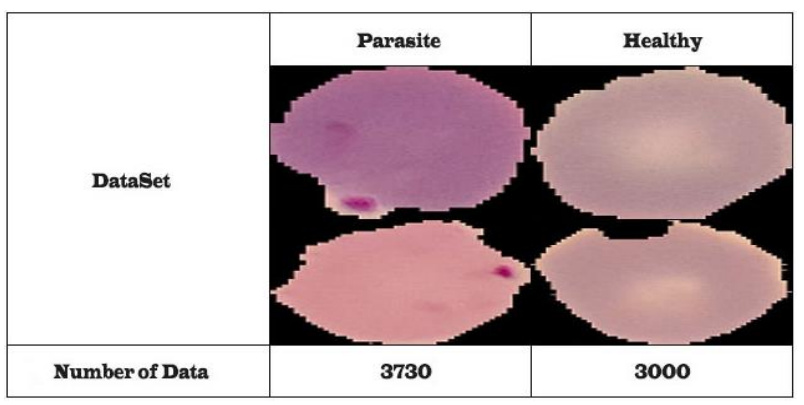

Figure 3. Class samples from the dataset 


\subsection{Dataset}

In this paper, data are obtained from Kaggle dataset [19]. There are 2 types of data classes. These data are used to diagnose malaria. The data in the first class are non-parasitic and in the second class there are parasitic data. The data set contains 3730 parasitic data and 3000 healthy data. The data classes and data numbers in the database used are as in Figure 3.

\section{APPLICATION AND RESULTS}

The application was performed in Matlab environment and firstly the original data was classified with AlexNet, Resnet50, DenseNet201, Vgg19, GoogleNet and Inceptionv3 architectures. Confidence matrices of the classified data were obtained with accuracy values. After the original data was classified, Median filter and Gaussian filter were implemented to the data in Matlab environment [20]. The new filtered data is reclassified in ALexNet, ResnNet50, DenseNet201, Vgg19, GoogleNet and Inceptionv3 architectures. Confusion matrices and accuracy values were obtained with the classification process. Then the obtained values were compared with each other and the performances of the architectures were observed.

The main purpose of filtering images is to reduce noise without losing important information in the image. Noise can be caused by more than one factor. If a good noise filter is applied to different types of images, it is expected to give successful results. There are various filtering methods to reduce noise in the image. In this study, Gaussian filter and Median filter were used.

Table 1. AlexNet architecture test results

\begin{tabular}{|c|c|c|c|}
\hline \multicolumn{4}{|c|}{ AlexNet } \\
\hline & \multicolumn{3}{|c|}{ Confusion Matrix } \\
\hline \multirow{4}{*}{ Original Data } & & 1 & 2 \\
\hline & 1 & 0.7600 & 0.2400 \\
\hline & 2 & 0.0133 & 0.9867 \\
\hline & \multicolumn{3}{|c|}{ Accuracy: $\mathbf{8 7 . 3 3} \%$} \\
\hline \multirow{4}{*}{ Data with Gauss Filter } & & 1 & 2 \\
\hline & 1 & 0.9533 & 0.0667 \\
\hline & 2 & 0.0233 & 0.9767 \\
\hline & \multicolumn{3}{|c|}{ Accuracy: $96.50 \%$} \\
\hline \multirow{4}{*}{ Data with Median Filter } & & 1 & 2 \\
\hline & 1 & 0.9167 & 0.0833 \\
\hline & 2 & 0.0933 & 0.9067 \\
\hline & \multicolumn{3}{|c|}{ Accuracy: 91.17\% } \\
\hline
\end{tabular}

Generally, Gaussian filter is one of the most preferred types of linear filters. Since the Gaussian filter mask is detachable, filtering can be performed faster. The output of the Gaussian filter is obtained by taking the weighted average of neighboring pixels. The distribution of weights in the Gaussian filter is determined by the two-dimensional Gaussian function [21].

The purpose of the Median filter is to decrease the hard tone changes in the image and make the image softer [22, 23]. The results obtained in the AlexNet model are given in Table 1.

AlexNet architecture has obtained $87.33 \%$ accuracy in original data. after filtering to the data set, they gained an accuracy rate of $95.50 \%$ in the Gauss filter and $91.17 \%$ in the Median Filter. AlexNet achieved the maximum accuracy in
Gauss filtered data. Original data was obtained with the lowest accuracy rate of $87.33 \%$.

The results obtained in the Resnet50 model are given in Table 2.

Table 2. ResNet50 architecture test results

\begin{tabular}{|c|c|c|c|}
\hline \multicolumn{4}{|c|}{ Resnet50 } \\
\hline & \multicolumn{3}{|c|}{ Confusion Matrix } \\
\hline \multirow{4}{*}{ Original Data } & & 1 & 2 \\
\hline & 1 & 0.8667 & 0.1333 \\
\hline & 2 & 0.0100 & 0.9900 \\
\hline & \multicolumn{3}{|c|}{ Accuracy: $\mathbf{9 2 . 8 3} \%$} \\
\hline \multirow{4}{*}{ Data with Gauss Filter } & & 1 & 2 \\
\hline & 1 & 0.9700 & 0.0300 \\
\hline & 2 & 0.0700 & 0.9300 \\
\hline & \multicolumn{3}{|c|}{ Accuracy: $\mathbf{9 5 . 0 0} \%$} \\
\hline \multirow{4}{*}{ Data with Median Filter } & & 1 & 2 \\
\hline & 1 & 0.9100 & 0.0900 \\
\hline & 2 & 0.0700 & 0.9300 \\
\hline & \multicolumn{3}{|c|}{ Accuracy: $\mathbf{9 2 . 0 0} \%$} \\
\hline
\end{tabular}

Resnet50 architecture has obtained $92.83 \%$ accuracy in original data. after filtering to the data set, they gained an accuracy rate of $95.00 \%$ in the Gauss filter and $92.00 \%$ in the Median Filter. Resnet50 achieved the maximum accuracy in Gauss filtered data. Original data was obtained with the lowest accuracy rate of $92.83 \%$.

The results obtained in the Densenet201 model are given in Table 3.

Table 3. DenseNet201 architecture test results

\begin{tabular}{|c|c|c|c|}
\hline \multicolumn{4}{|c|}{ DenseNet201 } \\
\hline & \multicolumn{3}{|c|}{ Confusion Matrix } \\
\hline \multirow{4}{*}{ Original Data } & & 1 & 2 \\
\hline & 1 & 0.9334 & 0.0666 \\
\hline & 2 & 0.0467 & 0.9533 \\
\hline & \multicolumn{3}{|c|}{ Accuracy: $\mathbf{9 4 . 3 3} \%$} \\
\hline \multirow{4}{*}{ Data with Gauss Filter } & & 1 & 2 \\
\hline & 1 & 0.9733 & 0.0267 \\
\hline & 2 & 0.0167 & 0.9833 \\
\hline & \multicolumn{3}{|c|}{ Accuracy: $\mathbf{9 7 . 8 3} \%$} \\
\hline \multirow{4}{*}{ Data with Median Filter } & & 1 & 2 \\
\hline & 1 & 0.8467 & 0.1533 \\
\hline & 2 & 0.0600 & 0.9400 \\
\hline & \multicolumn{3}{|c|}{ Accuracy: $\mathbf{8 9 . 3 3 \%}$} \\
\hline
\end{tabular}

Densenet201 architecture has obtained $94.33 \%$ accuracy in original data. after filtering to the data set, they gained an accuracy rate of $97.83 \%$ in the Gauss filter and $89.33 \%$ in the Median Filter. Densenet201 achieved the maximum accuracy in Gauss filtered data. Median filter data was obtained with the lowest accuracy rate of $89.33 \%$.

The results obtained in the Vgg19 model are given in Table 4.

Vgg19 architecture has obtained $85.67 \%$ accuracy in original data. after filtering to the data set, they gained an accuracy rate of $94.00 \%$ in the Gauss filter and $87.50 \%$ in the Median Filter. Vgg19 achieved the maximum accuracy in Gauss filtered data. Original data was obtained with the lowest accuracy rate of $85.67 \%$.

The results obtained in the GoogleNet model are given in Table 5. 
Table 4. Vgg19 architecture test results

\begin{tabular}{|c|c|c|c|}
\hline \multicolumn{4}{|c|}{ Resnet50 } \\
\hline & \multicolumn{3}{|c|}{ Confusion Matrix } \\
\hline \multirow{4}{*}{ Original Data } & & 1 & 2 \\
\hline & 1 & 0.9933 & 0.0067 \\
\hline & 2 & 0.2800 & 0.7200 \\
\hline & \multicolumn{3}{|c|}{ Accuracy: $\mathbf{8 5 . 6 7 \%}$} \\
\hline \multirow{4}{*}{ Data with Gauss Filter } & & 1 & 2 \\
\hline & 1 & 0.9100 & 0.0900 \\
\hline & 2 & 0.0300 & 0.9700 \\
\hline & \multicolumn{3}{|c|}{ Accuracy: $\mathbf{9 4 . 0 0 \%}$} \\
\hline \multirow{4}{*}{ Data with Median Filter } & & 1 & 2 \\
\hline & 1 & 0.8567 & 0.1433 \\
\hline & 2 & 0.1067 & 0.8933 \\
\hline & \multicolumn{3}{|c|}{ Accuracy: $\mathbf{8 7 . 5 0} \%$} \\
\hline
\end{tabular}

Table 5. GoogleNet architecture test results

\begin{tabular}{|c|c|c|c|}
\hline \multicolumn{4}{|c|}{ GoogleNet } \\
\hline & \multicolumn{3}{|c|}{ Confusion Matrix } \\
\hline \multirow{4}{*}{ Original Data } & & 1 & 2 \\
\hline & 1 & 0.9300 & 0.0700 \\
\hline & 2 & 0.0800 & 0.9200 \\
\hline & \multicolumn{3}{|c|}{ Accuracy: $\mathbf{9 2 . 5 0} \%$} \\
\hline \multirow{4}{*}{ Data with Gauss Filter } & & 1 & 2 \\
\hline & 1 & 0.8933 & 0.1067 \\
\hline & 2 & 0.0267 & 0.9733 \\
\hline & \multicolumn{3}{|c|}{ Accuracy: $\mathbf{9 3 . 3 3} \%$} \\
\hline \multirow{4}{*}{ Data with Median Filter } & & 1 & 2 \\
\hline & 1 & 0.8167 & 0.1833 \\
\hline & 2 & 0.0867 & 0.9133 \\
\hline & \multicolumn{3}{|c|}{ Accuracy: $\mathbf{8 6 . 5 0} \%$} \\
\hline
\end{tabular}

GoogleNet architecture has obtained $92.50 \%$ accuracy in original data. after filtering to the data set, they gained an accuracy rate of $93.33 \%$ in the Gauss filter and $86.50 \%$ in the Median Filter. GoogleNet achieved the maximum accuracy in Gauss filtered data. Median filter data was obtained with the lowest accuracy rate of $86.50 \%$.

The results obtained in the Inception $\mathrm{V} 3$ model are given in Table 6.

Table 6. InceptionV3 architecture test results

\begin{tabular}{|c|c|c|c|}
\hline \multicolumn{4}{|c|}{ Inceptionv3 } \\
\hline & \multicolumn{3}{|c|}{ Confusion Matrix } \\
\hline \multirow{4}{*}{ Original Data } & & 1 & 2 \\
\hline & 1 & 0.9333 & 0.0667 \\
\hline & 2 & 0.0667 & 0.9333 \\
\hline & \multicolumn{3}{|c|}{ Accuracy: $\mathbf{9 3 . 3 3} \%$} \\
\hline \multirow{4}{*}{ Data with Gauss Filter } & & 1 & 2 \\
\hline & 1 & 0.9167 & 0.0833 \\
\hline & 2 & 0.0267 & 0.9733 \\
\hline & \multicolumn{3}{|c|}{ Accuracy: $\mathbf{9 5 . 5 0} \%$} \\
\hline \multirow{4}{*}{ Data with Median Filter } & & 1 & 2 \\
\hline & 1 & 0.8033 & 0.1967 \\
\hline & 2 & 0.0400 & 0.9600 \\
\hline & \multicolumn{3}{|c|}{ Accuracy: $\mathbf{9 1 . 1 7 \%}$} \\
\hline
\end{tabular}

Inceptionv3 architecture has obtained $93.33 \%$ accuracy in original data. after filtering to the data set, they gained an accuracy rate of $94.50 \%$ in the Gauss filter and $88.17 \%$ in the Median Filter. Inceptionv3 achieved the maximum accuracy in Gauss filtered data. Median filter data was obtained with the lowest accuracy rate of $86.50 \%$.

Accuracy rates obtained in $\mathrm{CNN}$ architectures are given in Table 7.

Table 7. Accuracy table of all results

\begin{tabular}{|c|c|c|c|}
\hline & Original & Gauss & Median \\
\hline AlexNet & $87.33 \%$ & $96.50 \%$ & $91.17 \%$ \\
\hline ResNet50 & $92.83 \%$ & $95.00 \%$ & $92.00 \%$ \\
\hline DenseNet201 & $94.33 \%$ & $97.83 \%$ & $89.33 \%$ \\
\hline Vgg19 & $85.67 \%$ & $94.00 \%$ & $87.50 \%$ \\
\hline GoogleNet & $92.50 \%$ & $93.33 \%$ & $86.50 \%$ \\
\hline Inceptionv3 & $93.33 \%$ & $94.50 \%$ & $88.17 \%$ \\
\hline
\end{tabular}

\section{CONCLUSION}

In this paper, malaria images were classified using $\mathrm{CNN}$ methods, which are very popular in recent years. The application was implemented in Matlab environment and using AlexNet, ResNet50, DenseNet201, Vgg19, GoogleNet and Inceptionv3 models. First, the original data was classified into 6 different architectures and then the Gauss filter and Median filter were applied to the data set. After both filters, the dataset was again classified into the AlexNet, ResNet50, DenseNet201, Vgg19, GoogleNet and Inceptionv3 architectures. As a result, 6 different accuracy values were obtained in 6 different architectures for the original data, 6 different accuracy values with Gauss filter applied data and 6 different accuracy values with median filter applied data. The highest accuracy value was obtained from the Gauss filter images with $97.83 \%$ classification of DenseNet201 architecture. The accuracy of Gaussian filtered data increased significantly. Working with Gaussian filtered data increased our accuracy when classifying. More successful results were obtained with Gaussian filter applied data. Once malaria data is classified, it will be easier to draw conclusions and diagnose the disease by specialists.

\section{REFERENCES}

[1] Sivaramakrishnan, R., Antani, S.K., Jaeger, S. (2017). Visualizing deep learning activations for improved malaria cell classification. Proceedings of The First Workshop Medical Informatics and Healthcare (MIH 2017), Halifax, Canada, pp. 40-47.

[2] Rajaraman, S., Jaeger, S., Antani, S.K. (2019). Performance evaluation of deep neural ensembles toward malaria parasite detection in thin-blood smear images. Peer J, 7: e6977. https://doi.org/10.7717/peerj.6977

[3] Quinn, J.A., Nakasi, R., Mugagga, P.K., Byanyima, P., Lubega, W., Andama, A. (2016). Deep convolutional neural networks for microscopy-based point of care diagnostics. 2016 Machine Learning and Healthcare Conference (MLHC 2016), Los Angeles, CA, pp. 271281.

[4] Shen, H., Pan, W.D., Dong, Y.H., Alim, M. (2016). Lossless compression of curated erythrocyte images using deep autoencoders for malaria infection diagnosis. In 2016 Picture Coding Symposium (PCS), Nuremberg, Germany, pp. https://doi.org/10.1109/PCS.2016.7906393 
[5] Vijayalashmi, A., Rajesh Kana, B. (2019). Deep learning approach to detect malaria from microscopic images. Multimedia Tools and Applications. https://doi.org/10.1007/s11042-019-7162-y

[6] Delahunt, C.B., Mehanian, C., Hu, L., McGuire, S.K., Champlin, C.R., Horning, M.P., Wilson, B.K., Thompson, C.M. (2015). Automated microscopy and machine learning for expert-level malaria field diagnosis. 2015 IEEE Global Humanitarian Technology Conference (GHTC), Seattle, WA, USA, pp. 393-399. https://doi.org/10.1109/GHTC.2015.7344002

[7] Bibin, D., Nair, M.S., Punitha, P. (2017). Malaria parasite detection from peripheral blood smear images using deep belief networks. IEEE Access, 5: 9099-9108. https://doi.org/10.1109/ACCESS.2017.2705642

[8] Rajaraman, S., Antani, S.K., Poostchi, M., Silamut, K., Hossain, M.A., Maude, R.J., Jaeger, S., Thoma, G.R. (2018). Pre-trained convolutional neural networks as feature extractors toward improved malaria parasite detection in thin blood smear images. Peer J, 6: e4568. https://doi.org/10.7717/peerj.4568

[9] Liu, Z., Li, J., Shen, Z., Huang, G., Yan, S., Zhang, C. (2017). Learning efficient convolutional networks through network slimming. In Proceedings of the IEEE International Conference on Computer Vision, pp. 27362744.

[10] Mao, H., Han, S., Pool, J., Li, W., Liu, X., Wang, Y., Dally, W.J. (2017). Exploring the regularity of sparse structure in convolutional neural networks. arXiv preprint arXiv:1705.08922.

[11] He, K., Zhang, X., Ren, S., Sun, J. (2016). Deep residual learning for image recognition. In Proceedings of the IEEE Conference on Computer Vision and Pattern Recognition, pp. 770-778.

[12] Haupt, J., Kahl, S., Kowerko, D., Eibl, M. (2018). Largescale plant classification using deep convolutional neural networks. In CLEF (Working Notes).

[13] Krizhevsky, A., Sutskever, I., Hinton, G.E. (2012). ImageNet classification with deep convolutional neural networks. Communications of the ACM, 60(6). https://doi.org/10.1145/3065386
[14] Szegedy, C., Liu, W., Jia, Y., Sermanet, P., Reed, S., Anguelov, D., Rabinovich, A. (2015). Going deeper with convolutions. In Proceedings of the IEEE Conference on Computer Vision and Pattern Recognition, pp. 1-9.

[15] Lin, C., Li, L., Luo, W., Wang, K.C., Guo, J. (2019). Transfer learning based traffic sign recognition using inception-v3 model. Periodica Polytechnica Transportation Engineering, 47(3): 242-250. https://doi.org/10.3311/PPtr.11480

[16] Yildirim, M., Çinar, A. (2019). Simultaneously realization of image enhancement techniques on realtime FPGA. In 2019 International Artificial Intelligence and Data Processing Symposium (IDAP), Malatya, Turkey, Turkey, $\quad$ pp. 1-6. https://doi.org/10.1109/IDAP.2019.8875959

[17] Simon, M., Rodner, E., Denzler, J. (2016). Imagenet pretrained models with batch normalization. arXiv preprint arXiv:1612.01452.

[18] Gatys, L.A., Ecker, A.S., Bethge, M. (2016). Image style transfer using convolutional neural networks. In Proceedings of the IEEE Conference on Computer Vision and Pattern Recognition, pp. 2414-2423.

[19] Kaggle: Your Machine Learning and Data Science Community. https://www.kaggle.com/datasets.

[20] MathWorks - Matlab \& Simulunk. https://www.mathworks.com/products/matlab.html.

[21] Kwon, Y., Kim, K.I., Tompkin, J., Kim, J.H., Theobalt, C. (2015). Efficient learning of image super-resolution and compression artifact removal with semi-local Gaussian processes. IEEE Transactions on Pattern Analysis and Machine Intelligence, 37(9): 1792-1805. https://doi.org/10.1109/TPAMI.2015.2389797

[22] Fan, W., Wang, K., Cayre, F., Xiong, Z. (2015). Median filtered image quality enhancement and anti-forensics via variational deconvolution. IEEE Transactions on Information Forensics and Security, 10(5): 1076-1091. https://doi.org/10.1109/TIFS.2015.2398362

[23] Yildirim, M., Çinar, A. (2019). Classification of white blood cells by deep learning methods for diagnosing disease. Revue d'Intelligence Artificielle, 33(5): 335-340. https://doi.org/10.18280/ria.330502 